\title{
Copper-zinc imbalance and renal tubular damage in a population of chronic environmental cadmium exposure
}

\author{
Tomoyuiki Kawada ${ }^{1}$
}

Received: 15 April 2020 / Accepted: 1 September 2020 / Published online: 9 September 2020

c) Springer-Verlag GmbH Germany, part of Springer Nature 2020

Keywords Copper-zinc imbalance $\cdot$ Renal tubular damage $\cdot$ Environmental cadmium exposure

Eom et al. (2020) conducted a cross-sectional study to assess the relationship between environmental cadmium $(\mathrm{Cd})$ exposure, copper-zinc imbalance and renal tubular damage in inhabitants living in a Cd-polluted area. The geometric mean of urinary Cd was $2.25 \mu \mathrm{g} / \mathrm{g}$ creatinine, and $2 \mu \mathrm{g} / \mathrm{g}$ creatinine was used for the cut-off point of binary $\mathrm{Cd}$ exposure classification. The risk of renal tubular damage was significantly associated with urinary $\mathrm{Cd}$ level, particularly in the lowest or highest serum copper-to-zinc ratio (CZR) tertile groups. I have some concerns about their study.

First, the authors cannot evaluate the causal association, and they presented the association between $\mathrm{Cd}$ exposure and renal tubular damage with special reference to the level of serum CZR.

Second, the authors used serum CZR as a determinant of oxidative stress markers. Oxidative stress is one of the causes for $\mathrm{Cd}$-induced nephrotoxicity. Cadmium is transported to renal proximal tubules by binding to specific substances, such as metallothioneins and glutathione. Thereafter, increase of reactive oxygen species (ROS) becomes a trigger of DNA damage and mitochondrial dysfunction, which will lead to apoptosis of renal proximal tubules. Unfortunately, there exist no clear evidence on the association between ROS, low levels Cd exposure and nephrotoxicity in human (Liu et al. 2009). Interactions of several heavy metals might exist in a Cd-polluted population, and relationship between CZR and several oxidative stress markers should be specified by further study.

Regarding the second query, I reported the multiple metal exposures and renal effect with special reference to their magnitude of contribution (Kawada 2014). I speculate that sex and age might contribute to the inter-relationship among Cd exposure, copper-zinc imbalance and renal tubular damage. Stratified analysis by sex and age is required to verify the association.

\section{References}

Eom SY et al (2020) Copper-zinc imbalance induces kidney tubule damage and oxidative stress in a population exposed to chronic environmental cadmium. Int Arch Occup Environ Health 93(3):337-344. https://doi.org/10.1007/s00420-019-01490-9

Kawada T (2014) Multiple metal exposures and renal effect: their magnitude of contribution. Occup Environ Med 71(2):154. https://doi. org/10.1136/oemed-2013-101698

Liu J, Qu W, Kadiiska MB (2009) Role of oxidative stress in cadmium toxicity and carcinogenesis. Toxicol Appl Pharmacol 238:209214. https://doi.org/10.1016/j.taap.2009.01.029

Publisher's Note Springer Nature remains neutral with regard to jurisdictional claims in published maps and institutional affiliations.
Tomoyuiki Kawada

kawada@nms.ac.jp

1 Department of Hygiene and Public Health, Nippon Medical School, 1-1-5 Sendagi, Bunkyo-ku, Tokyo 113-8602, Japan 